International Mathematical Forum, 1, 2006, no. 31, 1519 - 1528

\title{
Existence of Positive Solution for Some Nonlinear Second Order Boundary Value Problems
}

\author{
Zhanbing Bai \\ Institute of Mathematics, Shandong University of Science and Technology \\ Qingdao 266510, People's Republic of China \\ zhanbingbai@163.com \\ Mingfu Ma \\ Department of Applied Mathematics, University of Petroleum \\ Dongying 257061, People's Republic of China
}

Abstract. In this paper, we concentrate on existence of positive solution for the second-order differential equation boundary value problem

$$
\begin{gathered}
x^{\prime \prime}(t)+f\left(t, x(t), x^{\prime}(t)\right)=0, \quad 0<t<1, \\
x(0)=x(1)=0,
\end{gathered}
$$

where $f:[0,1] \times[0, \infty) \times R \rightarrow[0, \infty)$ is continuous. By using a new fixed point theorem of functional type in a cone, the existence of at least one positive solution is obtained. The emphasis is put on the nonlinear term $f$ is involved with the first-order derivative.

Keywords: Fixed point theorem; Boundary value problem; Positive solution.

Mathematics Subject Classification: 34B15

\section{INTRODUCTION}

In the past 20 years, there has been much attention focused on existence and multiplicity of positive solutions for diverse nonlinear ordinary differential equations, difference equations, and functional differential equations without dependence on the first order derivative, see [1] and the references therein. It 
is well known that the Guo-Krasnosel'skii's fixed point theorem [7, 9] in a cone play a extremely important role in above works.

Recently, Leggett-Williams' fixed point theorem [10], five functional fixed point theorem [2], the twin fixed point theorem [3] due to Avery and Henderson, and a generalization of Leggett-Williams fixed point theorem [4] due to Avery and Peterson, were obtained and applied to archive some new existence and multiplicity results.

However, all the above works were done under the assumption that the first order derivative is not involved explicitly in the nonlinear term. More recently, in [6] the authors given an application of Avery-Peterson fixed point theorem to obtain the multiplicity results. In [5], the authors established a new generalization of Leggett-Williams' fixed-point theorem. Both [5] and [6] are devoted to the multiplicity of positive solutions for the second order two-point boundary value problem

$$
\begin{gathered}
x^{\prime \prime}(t)+f\left(t, x(t), x^{\prime}(t)\right)=0, \quad 0<t<1, \\
x(0)=x(1)=0
\end{gathered}
$$

where $f:[0,1] \times[0, \infty) \times R \rightarrow[0, \infty)$ is continuous. In this paper, we establish a new fixed point theorem and apply it to obtain the existence of positive solutions for boundary value problem (1.1), (1.2). The emphasis is also put on the nonlinear term $f$ is involved with the first-order derivative explicitly.

\section{FiXed POINT THEOREM IN A CONE}

In this section, by the use of the theory of fixed point index we get a new fixed point theorem in a cone. For the definitions and properties of fixed point index, we refer the reader to $[7,9]$ for detains.

Let $X$ be a Banach space and $P \subset X$ a cone. Suppose $\alpha, \beta: X \rightarrow[0, \infty)$ are two continuous nonnegative functionals satisfying

$$
\alpha(\lambda x) \leq|\lambda| \alpha(x), \quad \beta(\lambda x) \leq|\lambda| \beta(x), \quad \text { for } x \in X, \lambda \in[0,1]
$$

and

$$
M_{1} \max \{\alpha(x), \beta(x)\} \leq\|x\| \leq M_{2} \max \{\alpha(x), \beta(x)\}, \quad \text { for } x \in X,
$$

where $M_{1}, M_{2}$ are two positive constants.

For $r, L>0$, let

$$
\Omega=\{x \in X \mid \alpha(x)<r, \beta(x)<L\}
$$


Then the second inequality of (2.2) implies that $\Omega$ is a bounded open set in $X$. In addition, setting

$$
\begin{aligned}
& C=\{x \in X \mid \alpha(x)=r, \beta(x) \leq L\}, \\
& D=\{x \in X \mid \alpha(x) \leq r, \beta(x)=L\},
\end{aligned}
$$

one has $\partial \Omega=C \cup D$.

Lemma 2.1. [7] Suppose $T: P \cap \bar{\Omega} \rightarrow P$ is a completely continuous operator. If

(i) $\inf _{x \in P \cap \partial \Omega}\|T x\|>0$;

(ii) $T x=\lambda x, x \in P \cap \Omega \Rightarrow \lambda \notin(0,1]$, then

$$
i(T, \Omega \cap P, P)=0 .
$$

Lemma 2.2. Suppose $T: P \cap \bar{\Omega} \rightarrow P$ is a completely continuous operator. If

(i) $\alpha(T x)<r$, for $x \in C \cap P$;

(ii) $\beta(T x)<L$, for $x \in D \cap P$, then

$$
i(T, \Omega \cap P, P)=1
$$

Proof. Obviously, $\partial \Omega \cap P=(C \cap P) \cup(D \cap P)$. Let

$$
h(\lambda, x)=\lambda T x, \quad \lambda \in[0,1], x \in \bar{\Omega} \cap P,
$$

then $h(\lambda, x):[0,1] \times(\bar{\Omega} \cap P) \rightarrow X$ is completely continuous. We claim that $h(\lambda, x) \neq x$ for $x \in \partial \Omega \cap P$ and $\lambda \in[0,1]$. Suppose by contrary that there exist $x_{0} \in \partial \Omega \cap P, \lambda_{0} \in[0,1]$ such that

$$
h\left(\lambda_{0}, x_{0}\right)=x_{0},
$$

then $x_{0}=\lambda_{0} T x_{0}$.

If $x_{0} \in C \cap P$, by the condition $(i)$, one has

$$
r=\alpha\left(x_{0}\right)=\alpha\left(\lambda_{0} T x_{0}\right) \leq \lambda_{0} \alpha\left(T x_{0}\right)<\lambda_{0} r \leq r
$$

a contradiction. On the other hand, if $x_{0} \in D \cap P$, with the use of the condition (ii),

$$
L=\beta\left(x_{0}\right)=\beta\left(\lambda_{0} T x_{0}\right) \leq \lambda_{0} \beta\left(T x_{0}\right)<\lambda_{0} L \leq L,
$$


a contradiction, too. It follows from the homotopy invariance and the normality of the fixed point index that

$$
\begin{aligned}
i(T, \Omega \cap P, P) & =i(h(1, x), \Omega \cap P, P) \\
& =i(h(0, x), \Omega \cap P, P) \\
& =i(\theta, \Omega \cap P, P)=1 .
\end{aligned}
$$

The proof is complete.

Lemma 2.3. Suppose $T: P \cap \bar{\Omega} \rightarrow P$ is a completely continuous operator. If

(i) $\alpha(T x)>r$, for $x \in C \cap P$;

(ii) $\beta(T x)>L$, for $x \in D \cap P$,

then

$$
i(T, \Omega \cap P, P)=0 .
$$

Proof. We claim that if $T x=\lambda x$ for some $x \in \partial \Omega \cap P$, then $\lambda \notin(0,1]$. Suppose by contrary that there exist $x_{0} \in \partial \Omega \cap P, \lambda_{0} \in[0,1]$ such that

$$
T x_{0}=\lambda_{0} x_{0} .
$$

If $x_{0} \in C \cap P$, by the condition $(i)$, one has

$$
r<\alpha\left(T x_{0}\right)=\alpha\left(\lambda_{0} x_{0}\right) \leq \lambda_{0} \alpha\left(x_{0}\right) \leq \alpha\left(x_{0}\right) \leq r,
$$

a contradiction. If $x_{0} \in D \cap P$, with the use of the condition $(i i)$, we have

$$
L<\beta\left(T x_{0}\right)=\beta\left(\lambda_{0} x_{0}\right) \leq \lambda_{0} \beta\left(x_{0}\right) \leq \beta\left(x_{0}\right) \leq L,
$$

a contradiction, too.

In addition, the first inequality of (2.2) and the assumptions of Lemma 2.3 implies

$$
\inf _{x \in P \cap \partial \Omega}\|T x\| \geq M_{1} \cdot \inf _{x \in P \cap \partial \Omega} \max \{\alpha(T x), \beta(T x)\} \geq M_{1} \cdot \max \{r, L\}>0 .
$$

Therefore, with the use of Lemma 2.1, we have

$$
i(T, \Omega \cap P, P)=0 .
$$

The proof is complete.

By using Lemma 2.2 and 2.3, we can easily prove the following main result.

Theorem 2.1. Let $r_{2}>r_{1}>0, L_{2}>L_{1}>0$ be constants and

$$
\Omega_{i}=\left\{x \in X \mid \alpha(x)<r_{i}, \beta(x)<L_{i}\right\}, \quad i=1,2
$$


two open subsets in $X$ such that $\theta \in \Omega_{1} \subset \bar{\Omega}_{1} \subset \Omega_{2}$. In addition, let

$$
\begin{aligned}
& C_{i}=\left\{x \in X \mid \alpha(x)=r_{i}, \beta(x) \leq L_{i}\right\}, \quad i=1,2 ; \\
& D_{i}=\left\{x \in X \mid \alpha(x) \leq r_{i}, \beta(x)=L_{i}\right\}, \quad i=1,2 .
\end{aligned}
$$

Assume $T: P \rightarrow P$ is a completely continuous operator satisfying

$\left(S_{1}\right) \quad \alpha(T x) \leq r_{1}, x \in C_{1} \cap P ; \quad \beta(T x) \leq L_{1}, x \in D_{1} \cap P$ $\alpha(T x) \geq r_{2}, x \in C_{2} \cap P ; \quad \beta(T x) \geq L_{2}, x \in D_{2} \cap P ;$

or

$\left(S_{2}\right) \quad \alpha(T x) \geq r_{1}, x \in C_{1} \cap P ; \quad \beta(T x) \geq L_{1}, x \in D_{1} \cap P ;$ $\alpha(T x) \leq r_{2}, x \in C_{2} \cap P ; \quad \beta(T x) \leq L_{2}, x \in D_{2} \cap P$,

then $T$ has at least one fixed point in $\left(\bar{\Omega}_{2} \backslash \Omega_{1}\right) \cap P$.

Proof. Without loss of generality, suppose condition $\left(S_{1}\right)$ holds. Clearly, we can suppose there doesn't exist a fixed point of $T$ on both $P \cap \partial \Omega_{1}$ and $P \cap \partial \Omega_{2}$, otherwise, the theorem has been proved. Thus, we have $\alpha(T x)<r_{1}$, for $x \in C_{1} \cap P$ and $\beta(T x)<L_{1}$, for $x \in D_{1} \cap P$, by Lemma 2.2,

$$
i\left(T, \Omega_{1} \cap P, P\right)=1 .
$$

Also, by Lemma 2.3, $\alpha(T x)>r_{2}$ for $x \in C_{2} \cap P$ and $\beta(T x)>L_{2}$ for $x \in D_{2} \cap P$ implies

$$
i\left(T, \Omega_{2} \cap P, P\right)=0 .
$$

Then, according to the additivity of the fixed point index, we have

$$
\begin{aligned}
i\left(T,\left(\Omega_{2} \backslash \bar{\Omega}_{1}\right) \cap P, P\right) & =i\left(T, \Omega_{2} \cap P, P\right)-i\left(T, \Omega_{1} \cap P, P\right) \\
& =-1 .
\end{aligned}
$$

Hence there exists at least one $x \in\left(\bar{\Omega}_{2} \backslash \Omega_{1}\right) \cap P$ such that $T x=x$. The proof is complete.

Remark 2.1. Theorem 2.1 can be regarded as a generalization of Guo- Krasnosel'skii's fixed point theorem of cone expansion and compression. In fact, choose $\alpha(x)=\beta(x)=\|x\|, L_{1}=r_{1}, L_{2}=r_{2}$, we can acquire Guo-Krasnosel'skii's fixed point theorem.

\section{Existence Results of POSITIVE SOlutions}

In this section, we give some applications of Theorem 2.1 to dwell upon the existence of positive solutions for the second order two-point boundary value 
problem

$$
\begin{aligned}
x^{\prime \prime}(t)+f\left(t, x(t), x^{\prime}(t)\right) & =0, \quad 0<t<1 \\
x(0)=x(1) & =0,
\end{aligned}
$$

where $f:[0,1] \times[0, \infty) \times R \rightarrow[0, \infty)$ is continuous.

Let $X=C^{1}[0,1]$ with $\|x\|=\max \left\{\max _{0 \leq t \leq 1}|x(t)|, \max _{0 \leq t \leq 1}\left|x^{\prime}(t)\right|\right\}$ be a Banach space, $P=\left\{x \in X\left|x(t) \geq 0, x(t) \geq \max _{0 \leq t \leq 1}\right| x(t) \mid / 4, t \in[1 / 4,3 / 4]\right\} \subset$ $X$ a cone. Define functionals

$$
\alpha(x)=\max _{0 \leq t \leq 1}|x(t)|, \quad \beta(x)=\max _{0 \leq t \leq 1}\left|x^{\prime}(t)\right|, \quad \text { for } x \in X .
$$

Then $\alpha, \beta: X \rightarrow[0, \infty)$ are two continuous nonnegative functionals such that $\|x\|=\max \{\alpha(x), \beta(x)\}$, and (2.1), (2.2) hold. Denote $G(t, s)$ the Green's function for boundary value problem

$$
\begin{aligned}
-x^{\prime \prime}(t) & =0, \quad 0<t<1, \\
x(0) & =x(1)=0,
\end{aligned}
$$

then

$$
G(t, s)= \begin{cases}t(1-s), & 0 \leq t \leq s \leq 1, \\ s(1-t), & 0 \leq s \leq t \leq 1,\end{cases}
$$

and $G(t, s) \geq 0$ for $0 \leq t, s \leq 1$.

Let

$$
\begin{gathered}
M=\max _{0 \leq t \leq 1} \int_{0}^{1} G(t, s) d s=\frac{1}{8}, \\
N=\max _{0 \leq t \leq 1} \int_{1 / 4}^{3 / 4} G(t, s) d s=\frac{3}{32}, \\
(T x)(t):=\int_{0}^{1} G(t, s) f\left(s, x(s), x^{\prime}(s)\right) d s .
\end{gathered}
$$

Problem (3.1), (3.2) has a solution $x=x(t)$ if and only if $x$ solves the operator equation

$$
x=T x .
$$

It is well know that $T: P \rightarrow P$ is completely continuous.

Theorem 3.1. Suppose there exist constants $r_{2}>r_{1}>0, L_{2}>L_{1}>0,1 / 2>$ $h \geq 0$ such that the following assumptions hold

$\left(A_{1}\right) \quad f(t, u, v) \geq \frac{r_{1}}{N}, \quad$ for $(t, u, v) \in\left[\frac{1}{4}, \frac{3}{4}\right] \times\left[\frac{r_{1}}{4}, r_{1}\right] \times\left[-L_{1}, L_{1}\right]$;

$\left(A_{2}\right) \quad f(t, u, v) \geq \frac{2 L_{1}}{1-2 h}, \quad$ for $(t, u, v) \in[h, 1-h] \times\left[0, r_{1}\right] \times\left[-L_{1}, L_{1}\right]$; 
$\left(A_{3}\right) \quad f(t, u, v) \leq \min \left\{\frac{r_{2}}{M}, 2 L_{2}\right\}, \quad$ for $(t, u, v) \in[0,1] \times\left[0, r_{2}\right] \times\left[-L_{2}, L_{2}\right]$. Then Problem (3.1), (3.2) has at least one positive solution $x$ such that

$$
r_{1} \leq \max _{0 \leq t \leq 1} x(t) \leq r_{2}, \quad \max _{0 \leq t \leq 1}\left|x^{\prime}(t)\right| \leq L_{2},
$$

or

$$
L_{1} \leq \max _{0 \leq t \leq 1}\left|x^{\prime}(t)\right| \leq L_{2}, \quad \max _{0 \leq t \leq 1} x(t) \leq r_{2} .
$$

Proof. Let

$$
\Omega_{i}=\left\{x \in X \mid \alpha(x)<r_{i}, \beta(x)<L_{i}\right\}, \quad i=1,2
$$

be two bounded open sets in $X$. In addition,

$$
\begin{aligned}
& C_{i}=\left\{x \in X \mid \alpha(x)=r_{i}, \beta(x) \leq L_{i}\right\}, \quad i=1,2 \\
& D_{i}=\left\{x \in X \mid \alpha(x) \leq r_{i}, \beta(x)=L_{i}\right\}, \quad i=1.2 .
\end{aligned}
$$

For $x \in C_{1} \cap P$, one has $x(t) \geq \alpha(x) / 4=r_{1} / 4$ for $t \in[1 / 4,3 / 4]$. By $\left(A_{1}\right)$ and $G(t, s) \geq 0$,

$$
\begin{aligned}
\alpha(T x) & =\max _{t \in[0,1]}\left|\int_{0}^{1} G(t, s) f\left(s, x(s), x^{\prime}(s)\right) d s\right| \\
& \geq \max _{t \in[0,1]} \int_{1 / 4}^{3 / 4} G(t, s) f\left(s, x(s), x^{\prime}(s)\right) d s \\
& \geq \max _{t \in[0,1]} \int_{1 / 4}^{3 / 4} G(t, s) \cdot \frac{r_{1}}{N} d s \\
& =\frac{r_{1}}{N} \cdot \max _{t \in[0,1]} \int_{1 / 4}^{3 / 4} G(t, s) d s=r_{1} .
\end{aligned}
$$

From the nonnegativity of $f$ and the Green's function $G$, we have $(T x)^{\prime \prime}(t) \leq$ 0 . That is to say $T x$ is concave on $[0,1]$, it follows that

$$
\max _{t \in[0,1]}\left|(T x)^{\prime}(t)\right|=\max \left\{\left|(T x)^{\prime}(0)\right|,\left|(T x)^{\prime}(1)\right|\right\} .
$$


Taking into account the condition $\left(A_{2}\right)$, for $x \in D_{1} \cap P$,

$$
\begin{aligned}
\beta(T x) & =\max _{t \in[0,1]}\left|(T x)^{\prime}(t)\right| \\
& =\max _{t \in[0,1]}\left|-\int_{0}^{t} s f\left(s, x(s), x^{\prime}(s)\right) d s+\int_{t}^{1}(1-s) f\left(s, x(s), x^{\prime}(s)\right) d s\right| \\
& =\max \left\{\int_{0}^{1}(1-s) f\left(s, x(s), x^{\prime}(s)\right) d s, \int_{0}^{1} s f\left(s, x(s), x^{\prime}(s)\right) d s\right\} \\
& \geq \frac{2 L_{1}}{1-2 h} \cdot \max \left\{\int_{h}^{1-h}(1-s) d s, \int_{h}^{1-h} s d s\right\} \\
& \geq \frac{2 L_{1}}{1-2 h} \cdot \frac{1-2 h}{2}=L_{1} .
\end{aligned}
$$

For $x \in C_{2} \cap P$, by $\left(A_{3}\right)$, one has

$$
\begin{aligned}
\alpha(T x) & =\max _{t \in[0,1]}\left|\int_{0}^{1} G(t, s) f\left(s, x(s), x^{\prime}(s)\right) d s\right| \\
& \leq \max _{t \in[0,1]} \int_{0}^{1} G(t, s) \cdot \frac{r_{2}}{M} d s \\
& =\frac{r_{2}}{M} \cdot \max _{t \in[0,1]} \int_{0}^{1} G(t, s) d s=r_{2} .
\end{aligned}
$$

For $x \in D_{2} \cap P$, by $\left(A_{3}\right)$, we have

$$
\begin{aligned}
\beta(T x) & =\max _{t \in[0,1]}\left|(T x)^{\prime}(t)\right| \\
& =\max _{t \in[0,1]}\left|-\int_{0}^{t} s f\left(s, x(s), x^{\prime}(s)\right) d s+\int_{t}^{1}(1-s) f\left(s, x(s), x^{\prime}(s)\right) d s\right| \\
& =\max \left\{\int_{0}^{1}(1-s) f\left(s, x(s), x^{\prime}(s)\right) d s, \int_{0}^{1} s f\left(s, x(s), x^{\prime}(s)\right) d s\right\} \\
& \leq 2 L_{2} \cdot \max \left\{\int_{0}^{1}(1-s) d s, \int_{0}^{1} s d s\right\} \\
& \leq 2 L_{2} \cdot \frac{1}{2}=L_{2} .
\end{aligned}
$$

Now, Theorem 2.1 implies there exists $x \in\left(\bar{\Omega}_{2} \backslash \Omega_{1}\right) \cap P$ such that $x=T x$. Namely, Problem (3.1), (3.2) has at least one positive solution $x$ such that

$$
r_{1} \leq \alpha(x) \leq r_{2}, \quad \beta(x) \leq L_{2},
$$

or

$$
L_{1} \leq \beta(x) \leq L_{2}, \quad \alpha(x) \leq r_{2}
$$


Namely,

or

$$
r_{1} \leq \max _{0 \leq t \leq 1} x(t) \leq r_{2}, \quad \max _{0 \leq t \leq 1}\left|x^{\prime}(t)\right| \leq L_{2}
$$

$$
L_{1} \leq \max _{0 \leq t \leq 1}\left|x^{\prime}(t)\right| \leq L_{2}, \quad \max _{0 \leq t \leq 1} x(t) \leq r_{2} .
$$

The proof is complete.

Finally, we give an example to check the main results obtained.

Example 1. Consider the boundary value problem

$$
\begin{gathered}
x^{\prime \prime}(t)+f\left(t, x(t), x^{\prime}(t)\right)=0,0<t<1, \\
x(0)=x(1)=0
\end{gathered}
$$

where

$$
f(t, u, v)= \begin{cases}64\left[e^{t}+u^{2}+\left(\frac{v}{3000}\right)^{3}\right], & u \leq 10 \\ 64\left[e^{t}+100+\left(\frac{v}{3000}\right)^{3}\right], & u>10 .\end{cases}
$$

Choosing $h=0, r_{1}=3, L_{1}=8, r_{2}=840, L_{2}=3400$, then all the assumptions of Theorem 3.1 are satisfied. Consequently, Problem (3.3), (3.4) has at least one positive solution $x$ such that

$$
3 \leq \max _{0 \leq t \leq 1} x(t) \leq 840, \max _{0 \leq t \leq 1}\left|x^{\prime}(t)\right| \leq 3400
$$

or

$$
8 \leq \max _{0 \leq t \leq 1}\left|x^{\prime}(t)\right| \leq 3400, \max _{0 \leq t \leq 1} x(t) \leq 840 .
$$

We note that results of $[2,3,4,7,8,9]$, for example, are not applicable to the example. In conclusion, we see that the nonlinear term is involved in the first-order derivative explicitly.

Acknowledgement. This work is sponsored by the National Nature Science Foundation of China (10371006) and the Doctoral Program Foundation of Education Ministry of China (1999000722).

\section{REFERENCES}

[1] R.P. Agarwal, D. O'Regan, and P.J.Y. Wong, Positive Solutions of Differential, Difference, and Integral Equations, Kluwer Academic Publishers, Boston, 1999.

[2] R.I. Avery, A generalization of the Leggett-Williams fixed point theorem, Math. Sci. Res. Hot-line 2 (1998) 9-14. 
[3] R.I. Avery and J. Henderson, Two positive fixed points of nonlinear operators on ordered Banach spaces, (preprint).

[4] R.I. Avery and A.C. Peterson, Three positive fixed points of nonlinear operators on ordered Banach spaces, Comput. Math. Appl. 42 (2001) 313-322.

[5] Z.B. Bai and W.G. Ge, Existence of three positive solutions for some second-order boundary value problems Comput. Math. Appl. 48 (2004) 699-707.

[6] Z.B. Bai, Y.F. Wang and W.G. Ge, Triple positive solutions for a class of two-point boundary-value problems, Electronic J. Differential Equations, 2004: 06 (2004) 1-8.

[7] D. Guo and V. Lakshmikantham, Nonlinear Problems in Abstract Cones, Academic Press, New York, 1988.

[8] S. Hu, Nonlinear elliptic boundary value problems via cone analysis, Nonl. Anal. T.M.A. 18 (1992) 713-730.

[9] M. Krasnosel'skii, Positive Solutions of Operator Equations, Noordhoff, Gronignen, 1964.

[10] R.W. Leggett and L.R. Williams, Multiple positive fixed points of nonlinear operators on ordered Banach spaces, Indiana Univ. Math. J. 28 (1979) 673-688.

\section{Received: November 30, 2005}

